\title{
DEVELOPMENT OF A METHOD FOR MEASURING THE ASTRONOMICAL AZIMUTH USING AN ELECTRONIC TOTAL STATION
}

Khanzadyan M. A.*, Mazurkevich A.V.

" All-Russian Research Institute of Physical, Technical and Radio Engineering

Measurements "(FSUE" VNIIFTRI") R. P. Mendeleevo, Moscow Region, Russian

Federation

\section{Annotation}

In this article, the method of measuring the astronomical azimuth using an electronic total station, which is not intended for performing astronomical work, is considered. A method of measuring the astronomical azimuth using a high-precision electronic total station has been developed. Studies have been carried out to establish the influence of the components of errors in measurements of astronomical azimuth, forming the total budget of the error of the developed methodology, which has been tested on the reference stationary complex of metrological support of azimuth measuring instruments (CMS AMI).

\section{Introduction}

Currently, the Russian industry has not produced specialized theodolites and goniometers for astronomical measurements for more than twenty years. The main reason for this decision is a significant reduction in the need for these devices. However, it should be taken into account that there is still a small need for this type of measurement at the present time [1]. The main way out of this situation is to maintain the existing fleet of astronomical measuring instruments in working condition and develop methods for performing astronomical measurements with existing modern geodetic measuring instruments that are not intended and not adapted for this type of work. Thus, today the task of developing a specialized method of astronomical measurements for modern highprecision total stations is urgent. In this regard, a method for measuring astronomical azimuths was developed and tested using an electronic total station on a stationary complex of metrological support for azimuth measuring instruments (CMS AMI).

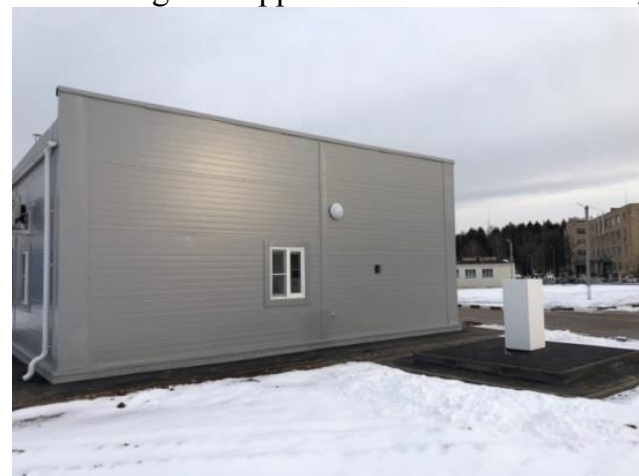

a)

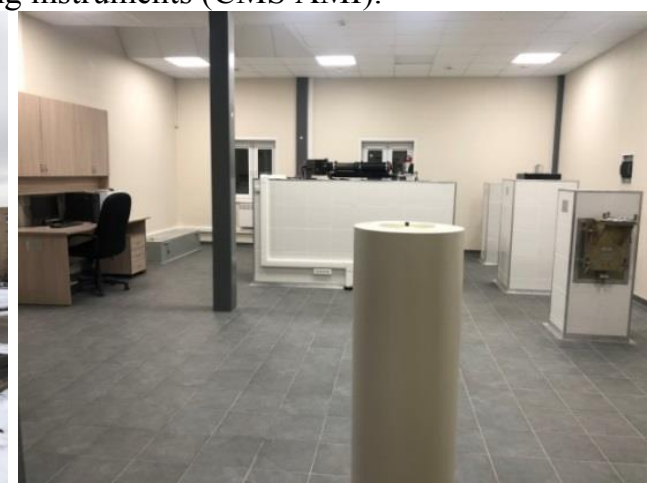

b)

Figure 1-General view of the Astropavillion building: a) external view of the astronomical laboratory b) General view of the azimuthal directions storage room of the astropavillion building

\footnotetext{
* Corresponding author: hma@vniiftri.ru
} 
CMS AMI was developed by specialists of FSUE "VNIIFTRI" in 2020 to ensure the uniformity of measurements for determining the azimuths of the initial directions, storing these directions, as well as for transmitting the unit of astronomical azimuth from the initial directions to azimuth measuring instruments (Figures 1 and 2)

CMS AMI consists of two parts:

- laboratory complex, consists of an automatic astronomical azimuth orientation system (AAAOS) and an optical azimuth direction keeper (OADK) (Figure 2);

- an azimuthal polygon consisting of three fundamental astronomical and geodetic points located at a distance of 300-400 $\mathrm{m}$ from each other (Figure 3).

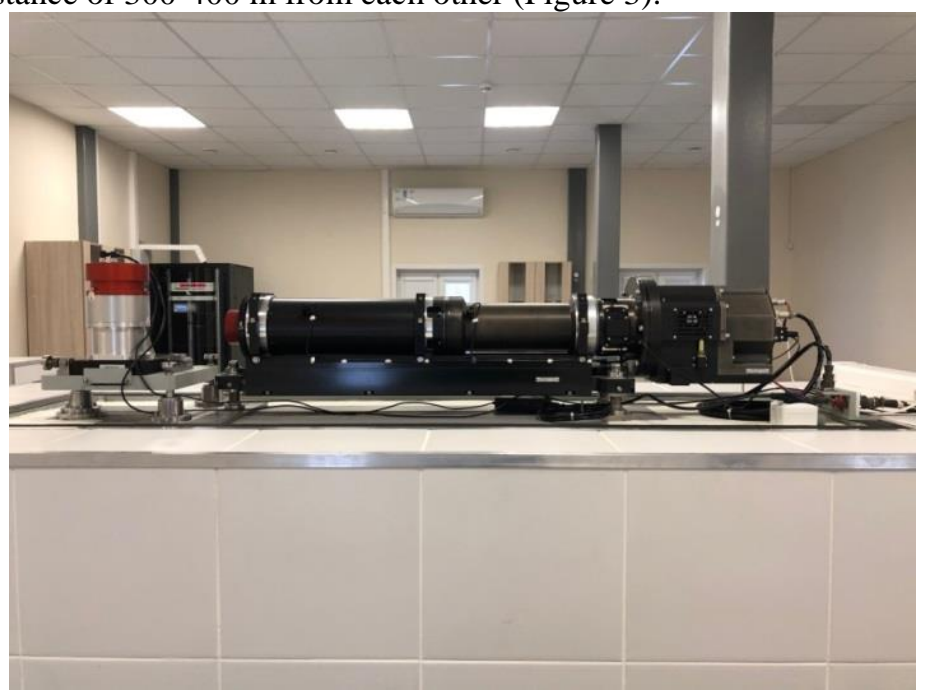

Figure 2-General view of the AAAOS

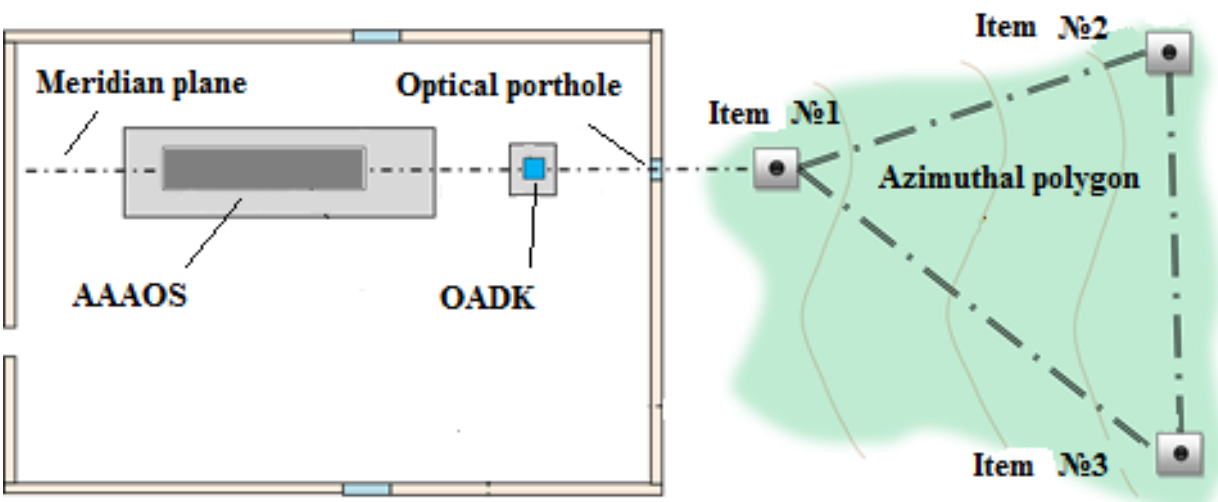

Figure 3-General view of the azimuth polygon

The principle of operation of the ASAAO is to determine the azimuth of the normal to the OHAN face from multiple observations of a set of stars culminating near the meridian on the matrix of the photodetector device of the television camera, as well as the identification of the position of stars according to the astronomical catalogs FK6, Hipparcos, PPMXL with the binding of the result to the UTC (SU) time scale using the 
state primary standard of the unit of time, frequency and the national time scale (GET 12018) [2] (Figure 4).

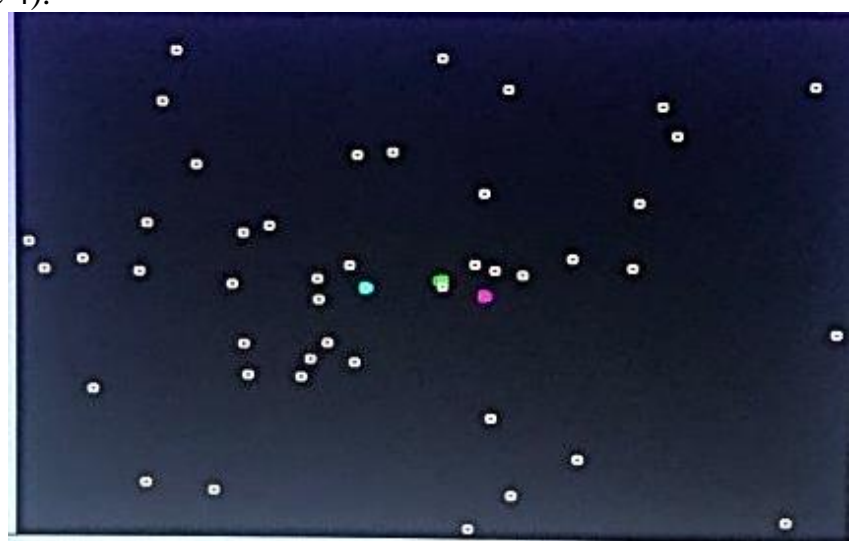

Figure 4-The number of visible stars on the AAAOS

The optical keeper of azimuthal directions is a four-sided mirror prism with certified values of the angles between the faces, mounted on the same optical axis with an astrovisir, designed to store the initial directions and transmit the azimuths of these directions to gyroscopic, astronomical and navigational azimuth measuring instruments (Figure 5).

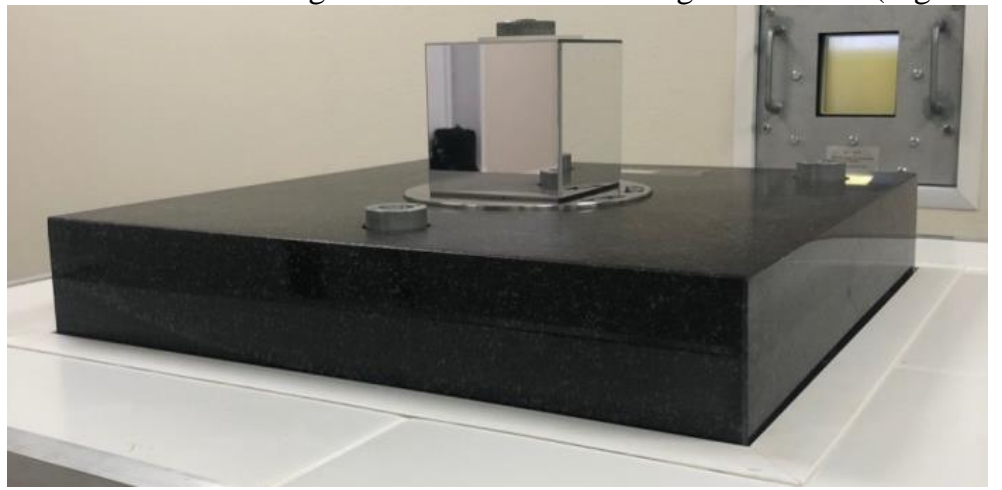

Figure 5 - The appearance of OADK

CMS AMI has the following metrological characteristics:

- non-excluded systematic error (NSE), no more than 0.5";

- the standard deviation (SD) of the measurement results of the astronomical azimuth on the OADK prism, not more than $0.5 "$.

\section{Measurement methodology}

In accordance with the metrological characteristics of CMS AMI, the following composition of the main and auxiliary means of measuring astronomical azimuth was developed. In the developed methodology, the Leica TS 60 I electronic total station and auxiliary equipment consisting of are used as the main means of measuring the astronomical azimuth: The Leica GS16 GNSS receiver is designed to provide time synchronization during astronomical measurements; a diagonal nozzle on the eyepiece is designed for angular measurements at high zenith distances; an autocollimation nozzle designed for pointing at the mirror face of an OADK optical prism (Figure 6) [3]. 


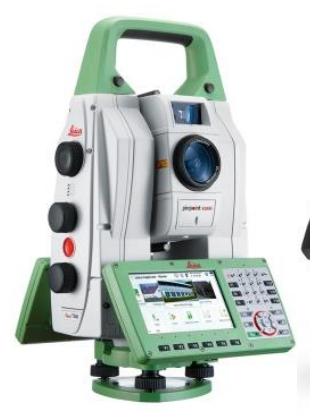

a)

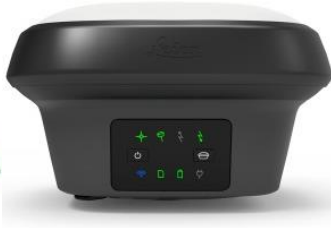

b)

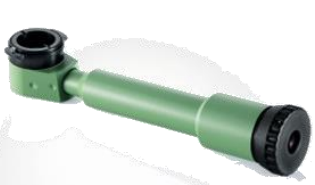

c)

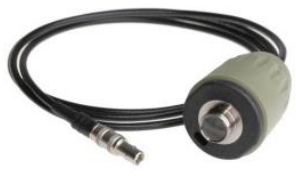

d)

Figure 6-The composition of the equipment used:

a) electronic total station Leica TS 60 I; b) GNSS receiver Leica GS 16;

c) diagonal nozzle on the GFZ3 eyepiece; d) autocollimation nozzle

The electronic total station is installed on an astronomical point located next to the autopavillon in the alignment that provides autocollimation binding to the mirror face of the OADK optical prism (Figure 8).

The electronic total station is synchronized with the GNSS receiver to link the time scale to the UTC (SU) time scale.

An autocollimation nozzle is installed on the eyepiece of the total station and measurements are made to the normal of the external mirror face of the OADK.

Then the autocollimation nozzle is changed to a diagonal one and the angular position of the Polar one is measured.

Then, in the built-in program of the total station, the measurement parameters are set by a full reception, according to the method of" Circular receptions", the measurement method (Polar- OADK -Polar- OADK) and the number of measured receptions (at least 25).

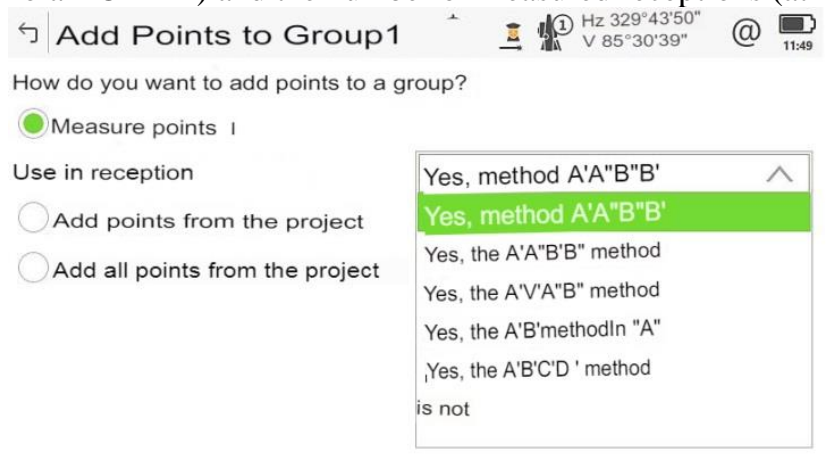

Figure 7-The window of the program "Method of circular techniques" in the electronic total station

The developed method allows using an electronic total station for measuring astronomical azimuth, and also has a significant advantage over the classical method of measuring astronomical azimuth using an astronomical theodolite, due to the automation of measurement actions. 


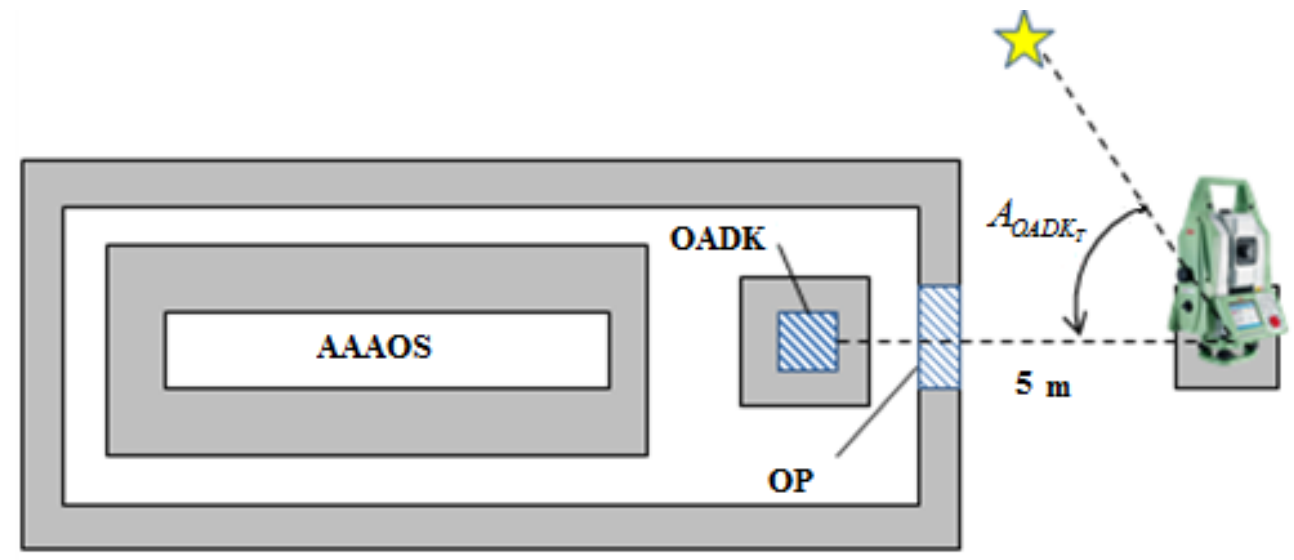

Figure 8-The scheme of measurements of the astronomical azimuth on OADK. (OP-

\section{Measurement results} optical porthole)

For the first time, work was carried out to assess the measurement error of the astronomical azimuth not only by the calculation method, but also with the help of the existing CMS AMI reference complex in FSUE VNIIFTRI.

The obtained measurements of the values of the astronomical azimuth of the direction to the outer face of the OADK were compared with the value of the azimuth obtained using the AAAOS from the composition of the CMS AMI.

An example of the measurement results is shown in Table 1.

Table 1-Measurement results

\begin{tabular}{|c|c|c|c|c|c|c|}
\hline № & Date & $\begin{array}{c}\text { Azimuth } \\
\text { OADK with } \\
\text { the help of } \\
\text { CMS AMI } \\
A_{O A D K_{A A A O S}}\end{array}$ & $\begin{array}{c}\text { Azimuth is } \\
\text { calculated } \\
\text { using an } \\
\text { electronic } \\
\text { total station } \\
A_{O A D K_{T}}\end{array}$ & $\begin{array}{c}\text { SD } \\
\sigma_{A_{O A D K_{A A A O S}}}\end{array}$ & $\begin{array}{c}\text { SD } \\
\sigma_{A_{O A D K_{T}}}\end{array}$ & $\begin{array}{c}\text { Error rate } \\
A_{O_{\text {OADK }}} \\
A_{O_{\text {OADK }}}\end{array}$ \\
\hline 1 & 10.12 .20 & $359^{\circ} 40^{\prime} 15.64^{\prime \prime}$ & $359^{\circ} 40^{\prime} 16.11^{\prime \prime}$ & $0.28^{\prime \prime}$ & $0.39^{\prime \prime}$ & $0.47^{\prime \prime}$ \\
\hline 2 & 25.01 .21 & $359^{\circ} 40^{\prime} 15.57^{\prime \prime}$ & $359^{\circ} 40^{\prime} 16.23^{\prime \prime}$ & $0.25^{\prime \prime}$ & $0.44^{\prime \prime}$ & $0.66^{\prime \prime}$ \\
\hline 3 & 17.02 .21 & $359^{\circ} 40^{\prime} 15.77^{\prime \prime}$ & $359^{\circ} 40^{\prime} 16.84^{\prime \prime}$ & $0.22^{\prime \prime}$ & $0.47^{\prime \prime}$ & $1.07^{\prime \prime}$ \\
\hline 4 & 11.03 .21 & $359^{\circ} 40^{\prime} 15.44^{\prime \prime}$ & $359^{\circ} 40^{\prime} 16.64^{\prime \prime}$ & $0.21^{\prime \prime}$ & $0.47^{\prime \prime}$ & $1.20^{\prime \prime}$ \\
\hline 5 & 29.03 .21 & $359^{\circ} 40^{\prime} 15.55^{\prime \prime}$ & $359^{\circ} 40^{\prime} 16.04^{\prime \prime}$ & $0.23^{\prime \prime}$ & $0.35^{\prime \prime}$ & $0.49^{\prime \prime}$ \\
\hline
\end{tabular}

The obtained result of the astronomical azimuth using an electronic total station differs by no more than 1.5 " from the azimuth obtained using the AAAOS installation, this is clearly evident from the results obtained, from which it follows that the developed technique is possible to use. However, in order to finally make sure that the developed methodology is working, it is necessary to continue the study in order to obtain more reliable data for a long period of time.

Using the obtained simultaneous measurements, it is possible to establish the sensitivity of the developed technique to the change in the position of the mirror face of the OADK prism over time, by plotting the dependence of the measurement result on the obtained 
measurement data (Figure 9). On the graph, you can clearly see the maximum movements of the mirror face of the OADK prism. The error of the developed method is higher than that of the standard CMS AMI complex, respectively, the maximum peaks on the graph have a more pronounced shape. Based on the analysis of the graphic data, we can conclude that the technique is well sensitive to external influencing factors that affect the change in the planned position over time of the mirror face of the OADK prism.

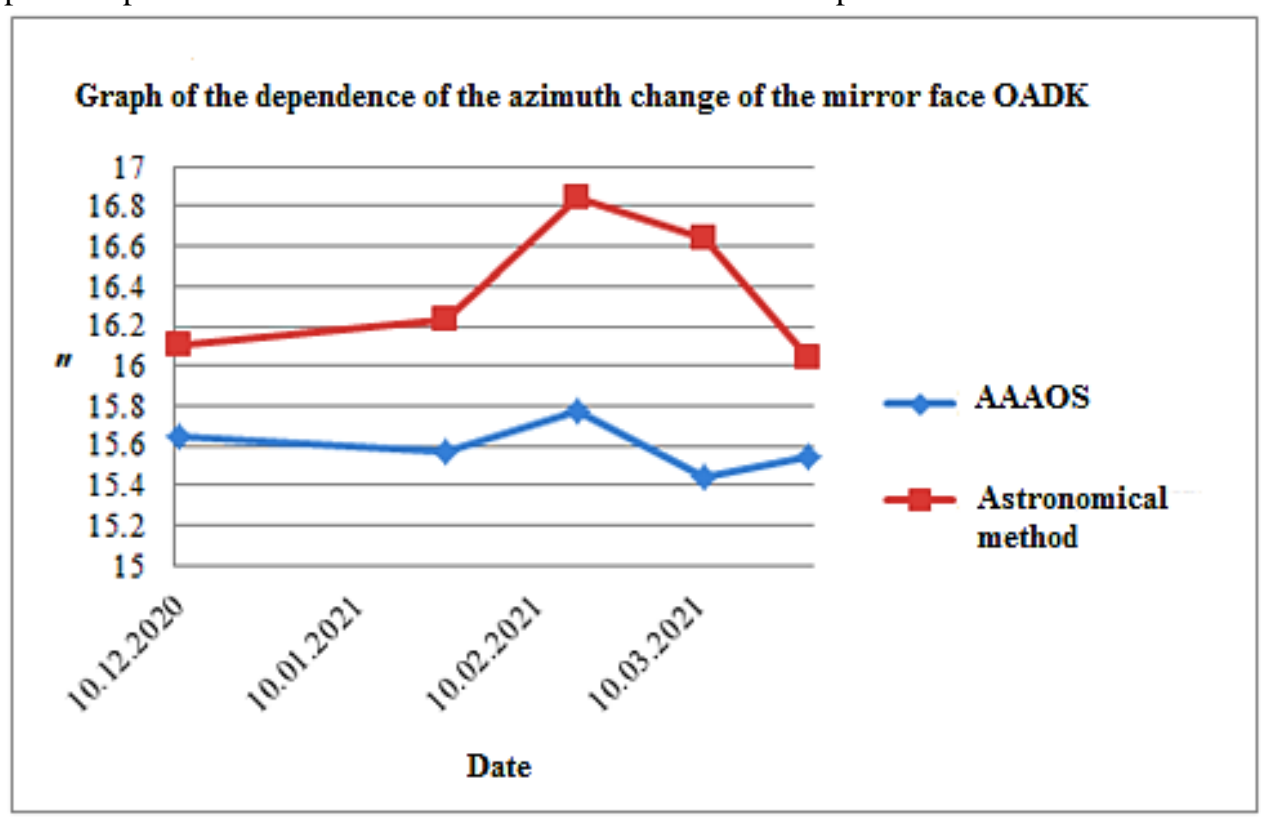

Figure 9-Graph of the dependence of the azimuth change of the mirror face of the OADK prism

For the developed and tested method, the analysis and calculation of random and systematic errors that have a total effect on the result of measurements of the astronomical azimuth are performed. The error of the method is represented as the limit of the permissible absolute error of measurements of the astronomical azimuth (with a confidence probability of 0.95 ) $[4,5]$.

The limits of the permissible absolute error of measurements of the astronomical azimuth (with a confidence probability of 0.95 ) are determined by the ratio:

$$
\Delta=K S_{\Sigma}
$$

where $K$ is the coefficient depending on the ratio of the random component of the error and the NSE.

The total standard deviation $S_{\Sigma}$ of the measurements of the astronomical azimuth is calculated by the formula:

$$
S_{\Sigma}=\sqrt{S_{\Theta}^{2}+S_{\dot{X}}^{2}}
$$

where $S_{\Theta}$ is the standard deviation of the NSE measurements of the astronomical azimuth

$$
S_{\Theta}=\frac{\Theta_{\Sigma}}{\sqrt{3}}
$$

where $\Theta$ is the boundary of the NSP measurements of the astronomical azimuth 


$$
\Theta_{\Sigma}= \pm k \sqrt{\Theta_{O A D K / S}^{2}+\Theta_{S}^{2}+\Theta_{F}^{2}+\Theta_{N}^{2}+\Theta_{D}^{2}}
$$

where $\Theta_{O A D K}$ is the error of sighting on the OADK prism; $\Theta_{S}$ is the error of sighting on the Polar Star ; $\Theta_{F}$ is the error for rolling the focusing device; $\Theta_{N}$ is the error caused by changing the nozzles (autocollimation to diagonal); $\Theta_{D}$ is the error caused by the longitudinal displacement of the beam passing through the optical porthole, taken from the passport value to the optical porthole; $\mathrm{k}$ is the coefficient determined by the selected confidence probability $\mathrm{P}=0.95$.

The error of sighting on the OADK prism and the Polar Star is determined by the formula:

$$
\Theta_{O A D K / S}=\frac{20 \prime \prime}{\Gamma^{\mathrm{x}}}
$$

where $\Gamma^{x}$ is the magnification of the optical tube of the electronic total station, 20" is the coefficient, the threshold of sensitivity of the human eye.

The error of the focusing device injection is obtained by the formula:

$$
\Theta_{F}=\frac{\left(\alpha_{2}-\alpha_{1}\right)}{2 S} \rho
$$

where $a_{1}, a_{2}$ are the readings on the scale of the AK-0.25 collimator relative to the vertical thread of the thread grid; $S$ is the distance from the total station to the scale equal to the distance to the OADK prism (Figure 8).

The error caused as a result of changing the nozzles is calculated by the formula:

$$
\Theta_{N}=\gamma_{2}-\gamma_{1}
$$

where $\gamma_{1}, \gamma_{2}$ are the values of the angles in seconds that characterize the deviations from the parallelism of the grid axes of the threads coming out of the eyepiece of the electronic total station due to the change of the eyepiece nozzle to a diagonal one

$$
\begin{aligned}
& \gamma_{1}=\alpha k_{1} \\
& \gamma_{1}=\alpha k_{2}
\end{aligned}
$$

where $\alpha$ is the price of dividing the scale of the collimator AK-0.25 in seconds; ; $k_{1}, k_{2}$ is the number of divisions corresponding to the displacement of the crosshair image from the center of the scale, respectively, in the horizontal and vertical planes.

The standard deviation of the arithmetic mean result $S_{\dot{X}}$ of the random component of the measurement error of the astronomical azimuth is calculated by the formula:

$$
S_{\dot{X}}=\frac{S}{\sqrt{n}}
$$

where $S$ is the standard deviation of the angle measurement equal to 0.5 " (from the metrological characteristics of the Leica TS 60 I total station); $n$ is the number of methods of measuring the angle of the astronomical azimuth.

$$
K=\frac{\varepsilon+\Theta_{\Sigma}}{S_{\dot{X}}+S_{\Theta}}
$$

where $\varepsilon$ are the confidence limits of the random error of measurements of the astronomical azimuth

$$
\varepsilon=t S_{\dot{X}}
$$

where $t$ is the Student's coefficient for a confidence probability of 0.95 .

Thus, according to formulas 1-12, the error of the developed methodology was calculated equal to $1.5^{\prime \prime}$ :

1. $\Delta=1,7 \cdot 0,9=1,5^{\prime \prime}$

2. $S_{\Sigma}=\sqrt{0,8^{2}+0,1^{2}}=0,9^{\prime \prime}$

3. $S_{\Theta}=\frac{1,5}{\sqrt{3}}=0,9^{\prime \prime}$

4. $\Theta_{\Sigma}= \pm 1,1 \sqrt{0,7^{2}+0,7^{2}+0,5^{2}+0,8^{2}+0,2^{2}}=1,5^{\prime \prime}$ 
5. $\Theta_{O A D K / S}=\frac{20 \prime \prime}{30^{\mathrm{x}}}=0,7^{\prime \prime}$

6. $\Theta_{F}=\frac{0,02^{\prime \prime}}{2 * 4000} * 206265^{\prime \prime}=0,5^{\prime \prime}$

7. $\Theta_{N}=0,9-0,1=0,8^{\prime \prime}$

8. $\gamma_{1}=0,01 * 10=0,1^{\prime \prime}$

9. $\gamma_{1}=0,01 * 90=0,9^{\prime \prime}$

10. $S_{\dot{X}}=\frac{0,5}{\sqrt{25}}=0,1^{\prime \prime}$

11. $K=\frac{0,2+1,5}{0,1+0,9}=1,7$

12. $\varepsilon=2,2 \cdot 0,1=0,2^{\prime \prime}$

\section{Conclusion}

Having analyzed the calculated measurement error of the developed methodology with the error obtained as a result of measurements (Table 1), it is possible to perform their comparative analysis by plotting a graph (Figure 10). In the graphical representation of the permissible and measured error, it can be concluded that the resulting error does not exceed the permissible one. In this regard, it can be assumed that the calculated values of the error components that affect the final error of the measurement result of the astronomical azimuth correspond to the real values of the measurement error.

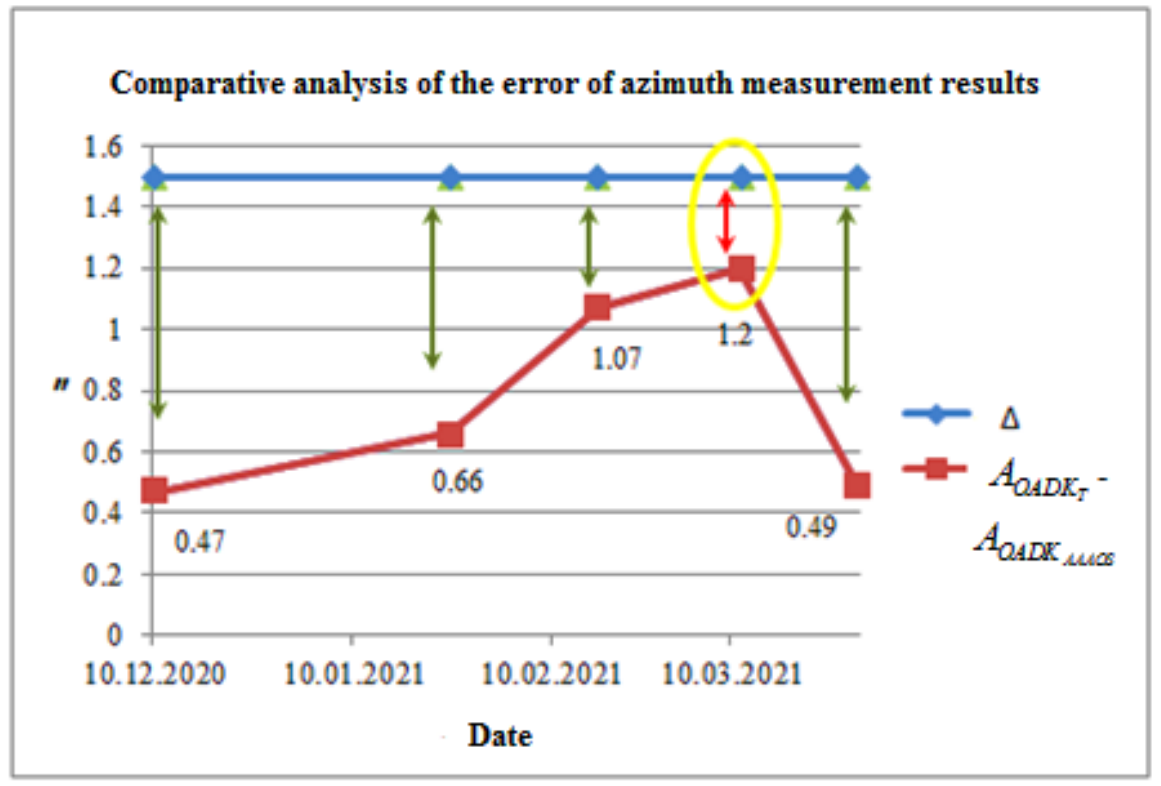

Figure 10-Graph of the dependence of the azimuth change of the mirror face of the OADK prism

\section{Conclusion}

Based on the results of testing the methodology and the error calculated for it, it can be assumed that the developed measurement method allows using modern measuring instruments for the measurement of astronomical azimuth and meets modern and promising requirements for the level of accuracy for existing types of activities. But the developed method has a number of disadvantages associated with the replacement of attachments, 
which adds an additional error to the measurement results, which cannot be excluded today due to the design features of the structure of electronic total stations.

\section{Literature}

1. Makhaev, A. Yu. Problematic issues of metrological support for testing and operation of an automatic system for determining the astronomical azimuth / A. Yu. Makhaev, S. B. Berkovich, N. I. Kotov, S. E. Chernyshev, N. V. Kuzmina/ Materials of the ninth AllRussian Scientific and Technical Conference Metrological support of Defense and security in the Russian Federation. Conference materials. - Pic. Povedniki, Moscow Region, October 23-25, 2012, pp. 92-95.

2. Makhaev, A. Yu. Automatic system for determining the astronomical azimuth /A. Yu. Makhaev, S. B. Berkovich, N. I. Kotov, S. V. Gaivoronsky, R. N. Sadekov, V. V. Tsodokova/ Metrology. Quarterly appendix to the scientific and technical journal "Measuring Equipment" - Moscow: Standartinform, 2015. No. 6. - pp. 11-20.

3. GKINP-01-153-81. A guide to astronomical definitions. Moscow "Nedra" 1984 p. $138-161$.

4. GOST 8.381-2009. Standards. Ways to express accuracy. - Instead of GOST 8.38180; Introduced from 01.01.12. - Moscow: FSUE "Standartinform", 2012;

5. GOST R 8.736-2011 State system for ensuring the uniformity of measurements (GSI). Direct multiple measurements. Methods of processing measurement results. The main provisions. 